\title{
HILBERT SPACES INDUCED BY HILBERT SPACE VALUED FUNCTIONS
}

\author{
SABUROU SAITOH \\ Dedicated to Professor Mitsuru Ozawa on his 60 th birthday
}

\begin{abstract}
Let $E$ be an arbitrary set and $\mathscr{F}(E)$ a linear space composed of all complex valued functions on $E$. Let $\mathcal{H}$ be a (possibly finite-dimensional) Hilbert space with inner product $(,)_{\mathscr{X}}$. Let $\mathbf{h}: E \rightarrow \mathfrak{K}$ be a function and consider the linear mapping $L$ from $\mathcal{H}$ into $\mathscr{F}(E)$ defined by $(\mathbf{F}, \mathbf{h}(p))_{\mathcal{K}}$. We let $\tilde{\mathcal{H}}$ denote the range of $L$. Then we assert that $\tilde{\mathcal{H}}$ becomes a Hilbert space with a reproducing kernel composed of functions on $E$, and, moreover, it is uniquely determined by the mapping $L$, in a sense. Furthermore, we investigate several fundamental properties for the mapping $L$ and its inverse.
\end{abstract}

1. Introduction. The author $[5,6]$ developed a general theory of integral transforms of Hilbert spaces and investigated miscellaneous concrete integral transforms by a unified method. The situation is as follows:

Let $d m$ denote a $\sigma$ finite positive measure. Let $L_{2}(\mathrm{dm})$ denote a usual separable Hilbert space composed of $d m$ integrable complex valued functions $F$ on a $d m$ measurable set $T$ and with finite norms $\|F\|_{L_{2}(d m)}^{2}=\int_{T}|F(t)|^{2} d m(t)$. For an arbitrary set $E$ and any fixed complex valued function $h(t, p)$ on $T \times E$ satisfying $h(t, p) \in L_{2}(d m)$ for any $p \in E$, we consider the integral transform of $F \in L_{2}(d m)$,

$$
f(p)=\int_{T} F(t) \overline{h(t, p)} d m(t),
$$

and we investigate this integral transform and its inverse. The basic method is based on the general theory of reproducing kernels using the direct integral theory established by Schwartz [7].

In this paper we show that we can develop by elementary methods the general theory [6] without the direct integral theory and, at the same time, with a much more general situation.

Further extensions of this paper will be considered in connection with the recent research of R. E. Curto and S. Salinas [4]. The refereee gave the author this point of view.

Let $E$ be an arbitrary set and $\mathscr{F}(E)$ a linear space compsed of all complex valued functions on $E$. Let $\mathcal{H}$ be a (possibly finite-dimensional) Hilbert space with inner

Received by the editors December 21, 1981 and, in revised form, September 16, 1982.

1980 Mathematics Subject Classification. Primary 44A05, 47A05; Secondary 30C40.

Key words and phrases. Hilbert space, linear mapping, linear operator, integral transform, reproducing kernel, isometry, completeness of functions. 
product $(,)_{\mathcal{H}}$. Let $\mathbf{h}: E \rightarrow \mathcal{H}$ be a function. Then we consider the linear mapping $L$ from $\mathcal{H}$ into $\mathscr{F}(E)$ defined by

$$
f(p)=(L \mathbf{F})(p)=(\mathbf{F}, \mathbf{h}(p))_{\mathcal{K}} .
$$

We let $\tilde{\mathcal{H}}$ denote the range of $L$. Then we show that $\tilde{\mathcal{H}}$ forms a Hilbert space admitting a reproducing kernel composed of functions on $E$, and, moreover, it is naturally induced from the mapping. Furthermore, we investigate several fundamental properties for the mapping $L$ and its inverse.

2. Construction of the range $\tilde{\mathcal{H}}$ of $L$. We first introduce the inner product $(,)_{\tilde{\mathcal{C}}}$ in $\tilde{\mathcal{H}}$ defined by

$$
\|f\|_{\tilde{\mathcal{C}}}=\inf \left\{\|F\|_{\mathcal{C}} ; f=L \mathbf{F}\right\}
$$

Then we obtain

THEOREM 2.1. [ $[\tilde{\mathbf{H}},(,) \tilde{\tilde{F}}]$ is a (possibly finite-dimensional) Hilbert space admitting the reproducing kernel $K(p, q)$ defined by

$$
K(p, q)=(\mathbf{h}(q), \mathbf{h}(p))_{\mathcal{X}} .
$$

Moreover, $L$ is an isometry between $\mathcal{H}$ and $\tilde{\mathcal{C}}$ if and only if $\{\mathbf{h}(p) ; p \in E\}$ is complete in $\mathcal{H}$.

Proof. From (1.2), null $(L)$ is a closed subspace in $\mathcal{H}$. Hence, for $f=L \mathbf{F}$, we have

$$
\|f\|_{\tilde{\mathcal{K}}}=\inf \left\{\|\mathbf{F}-\mathbf{G}\|_{\mathcal{K}} ; \mathbf{G} \in \operatorname{null}(L)\right\}=\left\|P_{\mathcal{G}} \mathbf{F}\right\|_{\mathcal{H}} \text {. }
$$

Here, $P_{\mathcal{G}}$ is an orthogonal projection from $\mathcal{H}$ onto $\mathcal{G}=\mathcal{H} \ominus$ null $(L)$. When we restrict $L$ on $\mathcal{G}$, then $\left.L\right|_{\mathcal{G}}$ is an isometry between $\left[\mathcal{G},(,)_{\mathcal{H}}\right]$ and $\left[\tilde{\mathcal{H}},(,)_{\mathcal{H}}\right]$, which implies that $\left[\tilde{\mathcal{H}},(,)_{\tilde{K}}\right]$ is a Hilbert space.

Next, we note that when $\mathbf{F} \in \operatorname{null}(L)$, then

$$
(\mathbf{F}, \mathbf{h}(p))_{\mathscr{K}}=0 \text { for all } p \in E .
$$

Hence, for any $q \in E, \mathbf{h}(q) \in \mathcal{G}$. From this fact we have, for any $f=L \mathbf{F}$,

$$
(f, K(\cdot, q))_{\tilde{H}}=(L \mathbf{F}, L \mathbf{h}(q))_{\tilde{K}}=\left(P_{\mathcal{G}} \mathbf{F}, P_{\mathcal{G}} \mathbf{h}(q)\right)_{\mathcal{H}}=(\mathbf{F}, \mathbf{h}(q))_{\mathcal{K}}=f(q),
$$

which implies that $K(p, q)$ is the reproducing kernel for $\tilde{\mathcal{H}}$.

In the last, we note that when $\{\mathbf{h}(p) ; p \in E\}$ is complete in $\mathcal{H}$, then $\mathcal{G}=\mathscr{H}$ so we have, for $f=L \mathbf{F}$,

$$
\|f\|_{\tilde{\mathcal{C}}}=\|\mathbf{F}\|_{\mathcal{K}}
$$

and vice versa. We thus complete the proof of Theorem 2.1.

3. Direct construction of the space $\tilde{\mathcal{H}}$. In Theorem 2.1 the norm in $\tilde{\mathcal{H}}$ is given in terms of the norm in $\mathcal{H}$ by making use of the mapping $L$. Here we note that we can construct $\tilde{\mathcal{H}}$ directly, in principle. This point of view is essentially important in dealing with concrete integral transforms. See $[5,6]$. In order to show this fact, we note that the function $K(p, q)$ on $E \times E$ is positive definite on $E$; that is,

$$
\sum_{i=1}^{n} \sum_{j=1}^{n} \alpha_{i} \overline{\alpha_{j}} K\left(p_{i}, p_{j}\right) \geqslant 0
$$


for any finite set $\left\{p_{i}\right\}$ of $E$ and for any complex numbers $\left\{\alpha_{i}\right\}$, as we see directly from (2.2). Then the theory of Moore and Aronszajn (see [1,2]) implies that for this $K(p, q)$ there exists a uniquely determined Hilbert space composed of functions on $E$ admitting $K(p, q)$ as the reproducing kernel. This space is just $\tilde{\mathcal{H}}$ stated.

We assume in the sequel that for $K(p, q)$, the space $\tilde{\mathcal{H}}$ can be realized in this sense. For miscellaneous concrete examples, see [6]. Then we have, of course, the following fundamental inequality for $L$ :

THEOREM 3.1. For the linear mapping

$$
f(p)=(\mathbf{F}, \mathbf{h}(p))_{\mathscr{K}},
$$

we obtain the inequality

$$
\|f\|_{\tilde{K}} \leqslant\|\mathbf{F}\|_{\mathscr{K}}
$$

When $\{\mathbf{h}(p) ; p \in E\}$ is complete in $\mathcal{H}$, then equality in (3.1) always holds.

Let $\left\{\mathbf{F}_{j}\right\}$ be an orthonormal basis for $\mathcal{H}$. Then

$$
\mathbf{h}(p)=\sum_{j}\left(\mathbf{h}(p), \mathbf{F}_{j}\right) \mathbf{F}_{j}=\sum_{j} \overline{f_{j}(p)} \mathbf{F}_{j} \quad \text { and } \quad \overline{\mathbf{h}(p)}=\sum_{j} f_{j}(p) \mathbf{F}_{j} .
$$

Thus $\overline{\mathbf{h}}=\Sigma_{j} f_{j}(\cdot) \mathbf{F}_{j}$. We define

$$
(f, \mathbf{h})_{\tilde{\mathfrak{K}}}=\sum_{j}\left(f, f_{j}\right)_{\tilde{\mathfrak{K}}} \mathbf{F}_{j}
$$

Then we obtain

THEOREM 3.2. We assume that for $f \in \tilde{\mathcal{H}}$,

$$
(f, \mathbf{h})_{\tilde{K}} \in \mathcal{H}
$$

and

$$
\left(f,(\mathbf{h}(q), \mathbf{h}(\cdot))_{\mathscr{K}}\right)_{\tilde{K}}=\left((f, \mathbf{h})_{\tilde{K}}, \mathbf{h}(q)\right)_{\mathscr{K}} \quad \text { for all } q \in E .
$$

Then we obtain the inequality

$$
\|f\|_{\tilde{\mathcal{K}}} \leqslant\left\|(f, \mathbf{h})_{\tilde{\mathcal{K}}}\right\|_{\mathscr{K}} .
$$

When $\{\mathbf{h}(p) ; p \in E\}$ is complete in $\mathcal{K}$, then equality always holds in (3.4).

Proof. We note that for any $q \in E$,

$$
f(p)=(f(\cdot), K(\cdot, p))_{\tilde{K} \mathcal{C}}=\left(f(\cdot),(\mathbf{h}(p), \mathbf{h}(\cdot))_{\mathscr{K}}\right)_{\tilde{K}}=\left((f, \mathbf{h})_{\tilde{K}}, \mathbf{h}(p)\right)_{\mathfrak{K}} .
$$

This shows that $f=L(f, \mathbf{h}) \tilde{\mathcal{K}}=L \mathbf{F}$, so by the definition of $\|\cdot\|_{\tilde{\mathscr{K}}}$ (see Theorem 3.1) we have $\|f\|_{\tilde{\mathcal{C}}} \leqslant\|(f, \mathbf{h}) \tilde{\mathcal{K}}\|_{\mathscr{\mathcal { C }}}$.

4. The inverse of $L$. We consider the inverse of $L$. Following Theorem 2.1, we need the assumption that $\{\mathbf{h}(p) ; p \in E\}$ is complete in $\mathcal{H}$. Then from the identity (3.5), we obtain

THEOREM 4.1. We assume (3.2) and (3.3) are valid, and $\{\mathbf{h}(p) ; p \in E\}$ is complete in $\mathcal{H C}$. Then for $f(p)=(\mathbf{F}, \mathbf{h}(p))_{\mathcal{S C}}$, we obtain its inverse

$$
\mathbf{F}=(f, \mathbf{h})_{\tilde{\mathcal{K}}} .
$$


When $\{\mathbf{h}(p) ; p \in E\}$ is not complete in $\mathcal{H}$, for $f$ we let $\mathbf{F}^{*}$ be the vector satisfying

$$
\|f\|_{\tilde{\mathcal{K}}}=\left\|\mathbf{F}^{*}\right\|_{\mathcal{H}}
$$

in (3.1). Then $\mathbf{F}^{*}$ is, of course, uniquely determined in $\mathcal{H}$. As to this vector $\mathbf{F}^{*}$, we obtain directly

THEOREM 4.2. We assume that (3.2) and (3.3) are valid and, further,

$$
\left(\mathbf{F}_{0},(f, \mathbf{h})_{\tilde{\mathcal{K}}}\right)_{\mathcal{H}}=\left(\left(\mathbf{F}_{0}, \mathbf{h}\right)_{\mathscr{H}}, f\right)_{\tilde{\mathcal{H}}} \quad \text { for all } \mathbf{F}_{0} \in \operatorname{null}(L) .
$$

Then we have

$$
\mathbf{F}^{*}=(f, \mathbf{h})_{\tilde{\mathcal{H}}} .
$$

As we see from concrete examples, conditions (3.2) and (3.3) are, in general, strong. For a more general and weak inverse formula in the case that the norm of $\tilde{\mathcal{H}}$ is realized by a positive measure, see $[5,6]$.

5. Generating functions and Hilbert space valued functions. We note that the starting point in our theory is, in fact, the identity (2.2). We now recall that there exists a general method which gives such an identity from a concrete Hilbert space $\tilde{\mathcal{H}}$ with the reproducing kernel $K(p, q)$ and an isometry mapping $\tilde{L}$ between $\tilde{\mathcal{H}}$ and $\mathcal{H}$, conversely. See Shapiro and Shields [8] and Burbea [3] for many concrete examples. The image of $K(\cdot, q)$ under this isometry $\tilde{L}$ is denoted by

$$
\mathbf{g}_{\tilde{L}}(q)=\tilde{L} K(\cdot, q),
$$

which is called "the generating vector of $\tilde{L}$ ". Then we have

$$
K(p, q)=(K(\cdot, q), K(\cdot, p))_{\tilde{K}}=(\tilde{L} K(\cdot, q), \tilde{L} K(\cdot, p))_{\mathcal{K}}=\left(\mathbf{g}_{\tilde{L}}(q), \mathbf{g}_{\tilde{L}}(p)\right)_{\mathfrak{K}},
$$

which is of type (2.2). From this identity and our theory, we obtain

THEOREM 5.1. For the linear mapping

$$
f(p)=\left(\mathbf{F}, \mathbf{g}_{\tilde{L}}(p)\right)_{\mathfrak{K}}, \quad \mathbf{F} \in \mathfrak{K},
$$

we have the identity

$$
\|f\|_{\tilde{K}}=\|\mathbf{F}\|_{: \mathcal{K}} .
$$

Further, the mapping (5.3) gives the isometry $\tilde{L}$ and the family of vectors $\left\{\mathbf{g}_{\tilde{L}}(p) ; p\right.$ $\in E\}$ is complete in $\mathcal{H}$.

Moreover, when for $f \in \tilde{\mathcal{H}}$,

$$
\left(f, \mathbf{g}_{\tilde{L}}\right)_{\tilde{\mathcal{K}}} \in \mathcal{H}
$$

and

$$
\left(f,\left(\mathbf{g}_{\tilde{L}}(q), \mathbf{g}_{\tilde{L}}\right)_{\mathcal{K}}\right)_{\tilde{\mathcal{K}}}=\left(\left(f, \mathbf{g}_{\tilde{L}}\right)_{\tilde{\mathcal{K}}}, \mathbf{g}_{\tilde{L}}(q)\right)_{\mathcal{H}} \quad \text { for all } q \in E
$$

are valid, then we obtain the inverse of (5.3),

$$
\mathbf{F}=\left(f, \mathbf{g}_{\tilde{L}}\right)_{\tilde{\mathcal{K}}} .
$$


Proof. We note that in (5.3), $\tilde{L} f=\mathbf{F}$ for any $\mathbf{F} \in \mathcal{H}$. Indeed, since $\tilde{L}$ is an isometry, for $\tilde{L} \tilde{f}=\mathbf{F}$, we have

$$
f(p)=\left(\mathbf{F}, \mathbf{g}_{\tilde{L}}(p)\right)_{\mathcal{K}}=(\tilde{L} \tilde{f}, \tilde{L} K(\cdot, p))_{\mathcal{H}}=(\tilde{f}(\cdot), K(\cdot, p))_{\tilde{K}}=\tilde{f}(p) .
$$

Hence, $f \equiv \tilde{f}$ and we see that (5.3) gives the isometry $\tilde{L}$. Of course, we have (5.4). Further, from (5.8), we see that $\left\{\mathbf{g}_{\tilde{L}}(p) ; p \in E\right\}$ is complete in $\mathcal{H}$. Moreover, from the argument in Theorem 3.2, we have the inverse formula (5.7).

For miscellaneous concrete examples, see $[5,6]$.

AcKnowledgments. The author wishes to thank Professors T. Ando, F. Beatrous, Jr., and the referee for their valuable advice and comments.

\section{REFERENCES}

1. N. Aronszajn, La théorie des noyaux reproduisants et ses applications. I, Proc. Cambridge Philos. Soc. 39 (1943), 118-153.

2. Theory of reproducing kernels, Trans. Amer. Math. Soc. 68 (1950), 337-404.

3. J. Burbea, Total positivity of certain reproducing kernels, Pacific J. Math. 68 (1976), 101-130.

4. R. E. Curto and N. Salinas, Generalized Bergman kernels and the Cowen-Douglas theory (preprint).

5. S. Saitoh, Integral transforms in Hilbert spaces (résumé), Proc. Japan Acad. 58 (1982), 361-364.

6. Integral transforms in Hilbert spaces (submitted).

7. L. Schwartz, Sous-espaces hilbertiens d'espaces vectoriels topologiques et noyaux associés (noyaux reproduisants), J. Analyse Math. 13 (1964), 115-256.

8. H. S. Shapiro and A. L. Shields, On the zeros of functions with finite Dirichlet integral and some related function spaces, Math. Z. 180 (1962), 229-249.

Department of Mathematics, Faculty of Engineering, Gunma University, Tenjin-Cho, Kiryu 376, JAPAN 